\title{
Glycogen Synthase Kinase 3 Inhibitors in the Next Horizon for Alzheimer's Disease Treatment
}

\author{
Ana Martinez, Carmen Gil, and Daniel I. Perez \\ Instituto de Química Médica, CSIC, Juan de la Cierva 3, 28006 Madrid, Spain \\ Correspondence should be addressed to Ana Martinez, amartinez@iqm.csic.es \\ Received 17 March 2011; Accepted 3 May 2011 \\ Academic Editor: Peter Crouch \\ Copyright () 2011 Ana Martinez et al. This is an open access article distributed under the Creative Commons Attribution License, \\ which permits unrestricted use, distribution, and reproduction in any medium, provided the original work is properly cited.
}

Glycogen synthase kinase 3 (GSK-3), a proline/serine protein kinase ubiquitously expressed and involved in many cellular signaling pathways, plays a key role in the pathogenesis of Alzheimer's disease (AD) being probably the link between $\beta$-amyloid and tau pathology. A great effort has recently been done in the discovery and development of different new molecules, of synthetic and natural origin, able to inhibit this enzyme, and several kinetics mechanisms of binding have been described. The small molecule called tideglusib belonging to the thiadiazolidindione family is currently on phase IIb clinical trials for AD. The potential risks and benefits of this new kind of disease modifying drugs for the future therapy of AD are discussed in this paper.

\section{Introduction}

After more than a century from its discovery and several decades of intensive research, the cause of Alzheimer's disease (AD) remains yet unknown [1]. As a consequence, it is very difficult to discover and/or develop effective therapies. For this reason, current therapies are mostly palliative and there exists a significant unmet medical need in the treatment of this devastating condition.

Different etiological hypotheses for $\mathrm{AD}$ origin have been considered in the last thirty years focused partially on the different histopathological lesions found in the brain of $\mathrm{AD}$ patients. The first one was the cholinergic hypothesis that considers the disease as a consequence of a deficit in the neurotransmitter acetylcholine. It is implicated in the cognitive process and consequently to its decline [2]. Although this strategy has severe limitations, drug research in this field has succeeded and today we have on the market four of the five drugs currently approved for the palliative treatment of $\mathrm{AD}$ based on drug research following this hypothesis [3]. The acetylcholinesterase inhibitors produced a slight enhancement in patient's cognitive abilities that partially improve temporarily their quality of life, but, in fact, the neurodegenerative disease does not stop [4].

In the eighties the amyloid cascade hypothesis emerged, and it was the most long considered theory [5]. It is based on the $\beta$-amyloid overproduction as responsible for the senile plaque formation and for the neurotoxicity that leads to the progressive neuronal death. However, controversial data about if $\beta$-amyloid is the cause of the disease or one of the main risk factors for $\mathrm{AD}$ are reported [6]. This dilemma is more pronounced recently because of the phase III clinical trials failure of the compounds discovered and developed based on amyloid hypothesis, such tramiprosate [7], tarenflurbil [8], and semagacestat [9].

The third postulated theory at the end of the past century was the tau-based hypothesis [10]. It is based on aberrant tau protein, a microtubule-associated protein that stabilizes the neuronal cytoskeleton, as the origin of $\mathrm{AD}$ pathology. As the time estimated to develop a new drug is around fifteen years, there is no time yet to see what happened with drugs specifically developed based on this hypothesis. Currently there are two phase IIb clinical trials with two different compounds, tideglusib and methylene blue. Both compounds have reported some positive results in the increase of cognitive level of $\mathrm{AD}$ patients after the first treatments on phase IIa clinical trials $[11,12]$. In the meanwhile, intensive research on the physiology and pathology of tau protein leads to the discovery of two kinases responsible for its posttranslational aberrant modifications. After cloning, these kinases were identified more than ten years ago, as the well-known glycogen synthase kinase 3 (GSK-3) and cyclin 
dependent kinase 5 (CDK-5), as the enzymes responsible for aberrant hyperphosphorylation of tau protein [13].

\section{The GSK-3 Hypothesis for AD}

In the last years a proposed alternative theory postulated that GSK-3 may play a more instigative role in the etiology of $\mathrm{AD}$, being the link between $\beta$-amyloid and tau protein [14]. It has been suggested that aberrant wnt or insulin signaling results in increased GSK-3 function, and this could be responsible for the observed hyperphosphorylation of tau and the formation of neurofibrillary tangles. This point is of utmost importance when growing evidence considered $\mathrm{AD}$ as diabetes type III, which means that a deficit of brain insulin signaling is the cause of $\mathrm{AD}$ [15]. In addition, elevated GSK-3 activity may induce increased $\beta$-amyloid formation through its action on $\gamma$-secretase and thereby give rise to the primary neuropathological lesion observed in $\mathrm{AD}$, the senile plaques [16]. GSK-3 has also been demonstrated to be involved in the mechanism underlying memory and learning, and dysregulation of the enzyme function may explain some of the early cognitive deficiencies observed in $\mathrm{AD}$ [17]. Moreover, overactivity of GSK-3 activates microglia through different cell signaling pathways involving, among others, $\mathrm{NF} \kappa \mathrm{B}$ and leading to the neuronal death [18]. Today there is no doubt about the GSK-3 upregulation in the brains of $\mathrm{AD}$ patients, and it is not clear if an overactivation and/or overexpression is the cause of its exacerbated activity [19]. All these observations point directly to GSK-3 as an excellent target to effectively treat all the clinical symptoms present in this devastating neurodegenerative disease. Currently, GSK-3 inhibitors represent promising disease modifying agents for $\mathrm{AD}$ neurodegeneration [20], a disease affecting the brain of the patients and the heart of their caregivers.

\section{GSK-3 Inhibitors as Drugs for Alzheimer's Disease: Risk versus Benefits}

GSK-3 is a proline-directed serine/threonine kinase. It affects the phosphorylation of a wide range of substrates and is involved in the regulation of many and diverse cellular functions, including metabolism, differentiation, proliferation, and apoptosis. GSK-3 is constitutively active, ubiquitous, and essential for life as it is demonstrated in many studies [21]. Its basal activity is regulated by many different mechanisms of action including phosphorylation at different residues (leading to inactive or superactive enzyme functions), proteinprotein interactions, and inhibition by endogenous peptides [22]. Our organism is prepared to restore, through compensative mechanism of action, a deficit in the expression and/or activity of the enzyme [23]. However, it is not able to downregulate GSK-3 with an endogenous alternative mechanism of action when this enzyme is exacerbated in different pathological conditions such as AD or diabetes type II. Thus, a smooth inhibition of GSK-3 able to restore levels of activity to physiological ones would be enough to produce an important therapeutic effect.
Moreover, GSK-3 is also known to play a key role in glucose metabolism and was first identified as the enzyme responsible for affecting the inhibitory phosphorylation of glycogen synthase (GS). This inhibitory effect leads to the reduction of rate conversion of glucose to glycogen, giving rise to elevations in blood glucose levels [24]. This function is controlled by insulin that through its binding to its receptor leads indirectly to activation of protein kinase B and subsequent phosphorylation of a key serine residue in the N-terminal domain of GSK-3 inactivating its function [25]. The major mechanism by which insulin stimulates the activity of GS in skeletal muscle is inactivation of GSK3. Insulin treatment causes about a 50\% inhibition of GSK-3 activity, a level sufficient to activate GS in skeletal muscle. Different experiments using ATP-competitive GSK3 inhibitors showed insulin sensitization without elevation of $\beta$-catenin levels. These data suggested that mild inhibition of GSK-3 (30-40\%) may be sufficient for insulin sensitization while a much more degree of inactivation $(>75 \%)$ of GSK-3 may be required for invoking growth-promoting effects [26].

An unrelated mechanism of GSK-3 inhibition operates in the wnt signaling cascade, a cellular pathway involved in controlling cell fate, differentiation, and proliferation. In this system, GSK-3 is complexed with APC, axin, and $\beta$-catenin, as well as other proteins [22]. When the wnt system is in a nonstimulated state, GSK-3 phosphorylates axin and APC, the effect of which is to create a more tightly associated complex. CK1, which is also associated with this protein assembly, performs an initial phosphorylation of $\beta$-catenin, and then, as priming substrate for GSK-3, it is hyperphosphorylated by this last enzyme resulting in its dissociation from the complex and its subsequent ubiquitination and destruction by proteasome. However, following binding of wnt ligands to their receptors, axin is displaced from the complex with GSK-3 as a result of binding of the latter to FRAT peptide, frequently rearranged in advanced Tcell lymphomas [27]. This leads to the dissociation of the destruction complex, the consequence of which is that $\beta$ catenin is no longer effectively phosphorylated and degraded. Cytosolic accumulation of $\beta$-catenin promotes its translocation to the nucleus where it binds to DNA and produces the transcription of certain oncogenes. This fact may raise the primary concern for GSK-3 targeted therapeutics and relate to the potential to induce transformation of nonmalignant cells or exacerbate preexisting malignancies through their actions on $\beta$-catenin. However, several in vivo experiments do not confirm this risk [28].

Apparently GSK-3 inhibition may be associated with significant mechanism-based toxicities, potentially ranging from hypoglycemia to tumorigenesis. However, it is worth mentioning that lithium has been used as standard therapeutic for the treatment of bipolar disorder since the 1950s. This agent is a weak inhibitor of GSK-3 that exerts its effect in vivo through a mixed mechanism of direct inhibition and activation of PKC- $\alpha$, the latter of which leads to increased GSK-3Ser9/21 phosphorylation, the inactivated enzyme state. At therapeutic doses, lithium is estimated to inhibit approximately a $25 \%$ of total GSK-3 activity, and this inhibition degree has not been associated with hypoglycemia, 

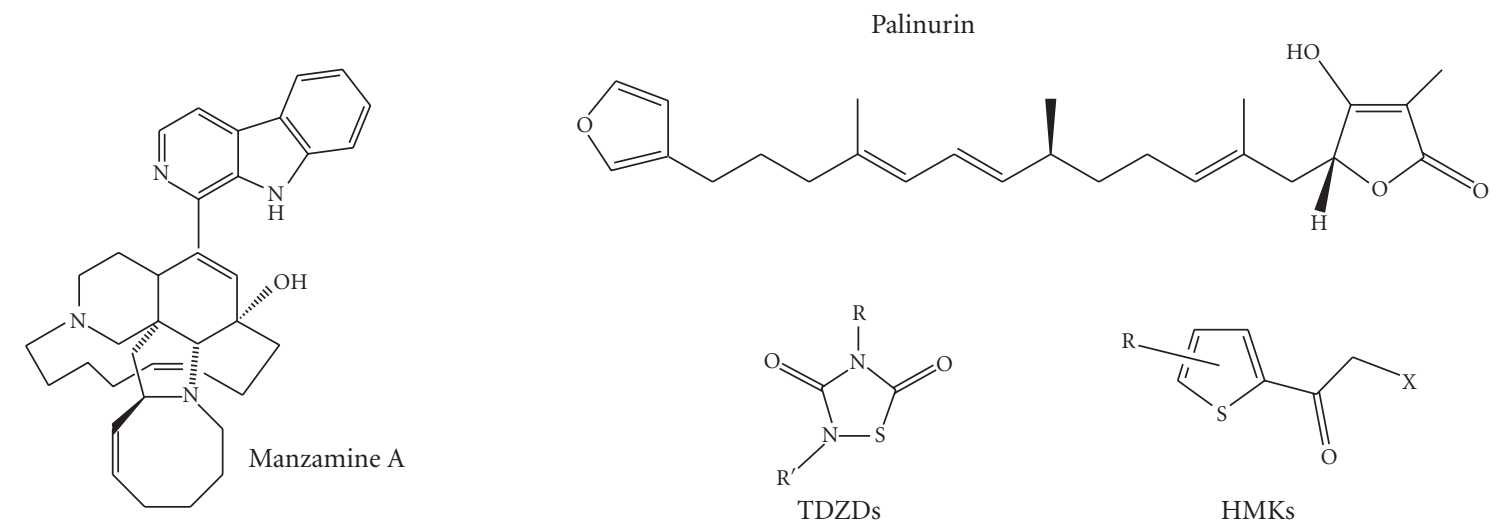

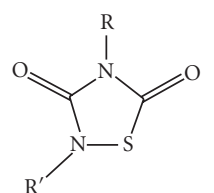

TDZDs

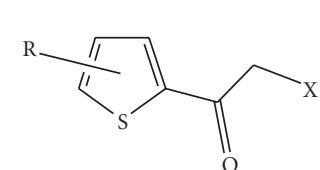

HMKs<smiles>CCCCCCCCCCCCCC(=O)NCC(=O)N[C@@H](CCCCN)C(=O)N[C@@H](CCC(=O)O)C(=O)N[C@@H](C)C(=O)N1C(=O)C2CCC(C(=O)N[C@@H](C)C(=O)N3CCC[C@H]3C(=O)N3C(=O)CCC3C(=O)N[C@@H](CCC(N)=O)C(=O)N[C@@H](COP(=O)(O)O)C(=O)N3CCC[C@H]3C(=O)O)N21</smiles>

Figure 1: non-ATP competitive GSK-3 inhibitors.

increased levels of tumorigenesis, or deaths from cancer. These epidemiological data are the most compelling argument for the potential safety of GSK-3 inhibitors, even considering that the final resulting in vivo GSK-3 inhibition through direct and indirect mechanism of action produced by the GSK-3 inhibitor treatment should be smooth and not greater than $25 \%$ of total activity. This means that, in pathological conditions, the GSK-3 inhibitor would be able to decrease the upregulation of the enzyme and, in the case that this treatment would slow down the GSK-3 physiological levels, other compensatory mechanisms of action would play the restorative function.

Another important challenge for a GSK-3 inhibitor as an $\mathrm{AD}$ treatment is its specific brain distribution. The drug needs to cross the blood-brain barrier to exert its action in the regulation of exacerbated GSK-3 brain levels. Usually this is not an easy task for any kind of drug, moreover when oral bioavailability is the preferred administration route for chronic $\mathrm{AD}$ treatment. It is very difficult to balance the equilibrium between molecular lipophilicity to enter into the brain and molecular hydrophilicity to be orally administrated, and this reason has ruled out several promising GSK-3 inhibitors from the race to the market. In addition, having suitable brain-to-plasma ratios may be determinant in achieving an adequate therapeutic index by limiting the peripheral exposure required to drive CNS efficacy. Once more, a smooth GSK-3 inhibitor is here required because in that case plasma levels of the GSK-3 inhibitor should not decrease more than $25 \%$ of physiological enzyme activity to avoid adverse and toxic effects.

\section{ATP versus Non-ATP Competitive GSK-3 Inhibitors as Drugs}

Over the past ten years a number of chemically diverse families of GSK-3 inhibitors have been discovered. They have been reviewed periodically $[29,30]$. The structural chemical diversity of compounds reported in this area has expanded considerably, especially in the patent literature, showing the great interest and expectation that this new kind of powerful drugs has arisen both in pharmaceutical and academic laboratories [31]. However, the great majority of these compounds compete with the ATP in their binding to GSK-3.

Human kinome is formed by more than 500 kinases with a more or less conserved site for binding the ATP, a common phosphate donor molecule used by all of them in their phosphorylating physiological function [32]. Thus, GSK-3 inhibitors that are non-ATP competitive are potentially attractive for several reasons, first of all because they may show better cellular and in vivo potency in comparison with competitive inhibitors having comparable absorption-distribution-metabolism-excretion (ADME) properties due to the absence of endogenous ATP competition. In addition, much better kinase selectivity may be expected from inhibitors that bind outside the ATP pocket. Moreover, this kind of kinase inhibitors should have lower values of $\mathrm{IC}_{50}$, which in the case of GSK-3 is not only beneficial but also necessary to avoid toxicity. Thus, non-ATP competitive GSK-3 inhibitors arise as the unique real potential drugs for the treatment of at least chronic diseases as $\mathrm{AD}$. 


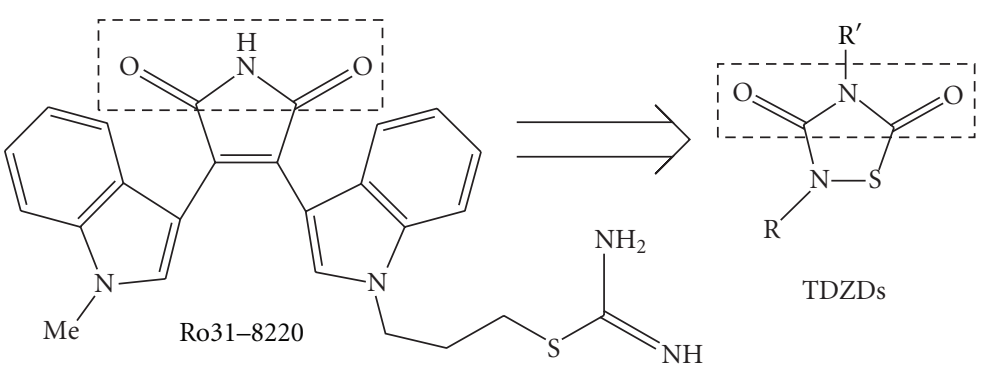

Figure 2: Common features between Ro31-8220 and TDZDs.

There are few GSK-3 inhibitor families reported with non-ATP competitive mechanism of action (Figure 1). The first one was the thiadiazolidindione family (TDZDs) [33]. TDZD-8, commercially available from different sources, has been one of the most useful pharmacological tools in the chemical genetic approach followed by many scientists to explore GSK-3 functions, as it is reflected in the great number of published manuscripts on the field using this small molecule $[34,35]$. More important for AD therapy, and probably for society, is tideglusib, a TDZD compound currently on phase IIb clinical trials for $\mathrm{AD}$ and the rare tauopathy called progressive supranuclear palsy (PSP).

In addition to TDZDs, two marine natural compounds, the alkaloid manzamine [36] and the sesquiterpene palinurin, have been reported as cell permeable non-ATP competitive GSK-3 inhibitors able to reduce tau phosphorylation in cell cultures [37]. The binding site of manzamine has been recently postulated by molecular modeling studies as an allosteric site at the back of ATP site, just in the junction of C-terminal and N-terminal globes of GSK-3, being the first GSK-3 allosteric modulator described [38]. At the moment, nothing is known about the potential binding site of palinurin although some quantitative structure activity relationship has been reported [39]. These compounds are valuable candidates, with privileged scaffolds provided by the nature, to be considered for further optimization of synthetic, biological, and ADME properties in a drug development process.

Another promising group of GSK-3 inhibitors is those specifically designed to interact with the priming substrate site present on GSK-3 [40]. The ability of GSK-3 for binding to prephosphorylated (priming) substrates and introduce a new phosphate group in the fourth amino acid from the priming site has been considered by Eldar-Finkelman group to provide substrate competitive GSK-3 inhibitors all of which are based on peptide scaffolds [41]. The small peptide L803-mts has proven to be effective in decreasing the early depressive behavior induced by mild traumatic brain injury [42] and in the improvement of glucose homeostasis in obese mice (ob/ob), an animal model of type II diabetes [43].

Finally, irreversible inhibitors of GSK-3 have just been reported representing a good alternative to avoid resistance in a future drug treatment as it is the case in other therapeutic areas as cancer where the therapy with protein kinase inhibitors is more advanced [44]. Halomethylketones (HMKs) are irreversible inhibitors with $\mathrm{IC}_{50}$ values in the low micromolar range, able to decrease tau phosphorylation in cell cultures, good kinase and neurotransmitter selectivity, and ability to cross the blood-brain barrier [45, 46]. GSK-3 inhibitor VII, commercially available, belongs to this class of compounds, and it is yet used in chemical genetic approaches for the study of GSK-3. Important is the fact that biophysical methods such mass spectrometry and the use of these last pharmacological tools have proven experimental data to show that Cys199 of GSK-3 is a key amino acid to modulate its activity [47], opening new opportunities for the future design of specific and valuables inhibitors.

\section{TDZDs: GSK-3 Inhibitors in Clinical Trials for AD}

As mentioned before, the TDZDs were the first non-ATP GSK-3 inhibitors reported in the literature [33]. The inhibitory kinase activity of TDZDs was discovered in a GSK-3directed program initiated in the late nineties in the Medicinal Chemistry Institute, CSIC. This activity was determined in a radiometric assay incubating together rabbit recombinant GSK-3 $\beta$ enzyme, the peptide derived from the sequence of the human glycogen synthase GS-1 as substrate, and $\left[\gamma_{-}{ }^{32} \mathrm{P}\right] \mathrm{ATP}$ in the appropriate buffer and temperature [48]. When the GSK-3 inhibition for the known PKC inhibitor Ro31-8220 was reported [49], we decided to include in our screening program some of the side products obtained in our laboratory in the synthesis of biological active compounds such as potassium channel openers [50], muscarinic agonist [51], or acetylcholinesterase inhibitors [52]. The main reason for this decision was the common features present in the chemical structure of TDZDs and Ro318220 , mainly the 1,3-dicarbonyl moiety in a five-membered ring with a nitrogen atom between both carbonyl groups (Figure 2). The results of the screening showed that three of the four compounds tested had an $\mathrm{IC}_{50}$, the compound concentration that inhibits $50 \%$ of the enzyme activity, in the low micromolar range [53]. After the discovery of TDZDs as GSK-3 inhibitors, different compounds with the maleimide core were reported as inhibitors of this versatile enzyme $[54,55]$, pointing out the relevance of this 1,3-dicarbonyl substitution joined by a nitrogen atom in a pentagonal ring for the interaction with GSK-3. Our extensive SAR studies showed that the thiadiazolidine scaffold is the best one to interact with GSK-3 [56]. 
All the work done in the hit to lead process and lead selection has been reported recently [57], and currently the clinical development of the most advanced candidate, called tideglusib and previously known as NP031112 or NP12 , is sponsored by a Spanish biotechnological company. In summer 2010, data from the phase II clinical trial of tideglusib were reported showing a trend in the cognition increase of the twenty mild to moderate $\mathrm{AD}$ patients treated for 24 weeks [58]. Now, clinical phase IIb trial is ongoing and the orphan drug status has been achieved for the development of tideglusib in the rare tauopathy PSP [59]. The recruitment of patients is closed and the results are expected by the end of this year.

In preclinical studies, the compound has shown an important neuroprotective effect using a kainate excitotoxicity model and i.c.v. and oral administration [60]. Moreover, very relevant for the future benchmark to clinical translation to $\mathrm{AD}$ patients are the results obtained in the chronic oral treatment for 3 months performed with tideglusib to the double transgenic APP $\times$ tau rodent model [61]. An increase in cognition using the Morris water maze test was observed after the treatment while all the histopathological features present on the mice brain related to $\mathrm{AD}$ pathology were reduced, such as the $\beta$-amyloid plaque load, the hyperphosphorylation of tau protein, the gliosis, and, what it is more important, the number of neuron deaths both in cortex and hippocampus [62]. Very interesting is the fact recently reported regarding the increase of insulin growth factor 1 (IGF-1) in mice brains, both in wild-type and APP $\times$ PS1 mice, after oral treatment with NP-12 for five days [63]. IGF1 is a potent neurotrophic peptide with therapeutic value for many neurodegenerative diseases including AD pathology [64]. These increased IGF-1 levels in mice would mean that there is not only a direct action of GSK-3 inhibitors in the brain and there is also an effect on some peripheral signalling pathways (as activation of the IGF-1 transporter megalin) that could be modified with this innovative treatment improving the $\mathrm{AD}$ pathology.

\section{Conclusions}

Clear evidence, both in cell and animal models and in $\mathrm{AD}$ brain patients, points to GSK-3 as one of the key players with a unique pivotal role in $\mathrm{AD}$ pathology. As the upregulation of the enzyme is the fatal cause for neurodegeneration, its inhibitors arise without any doubt as promising drugs for a future disease modifying treatment [65]. Some data suggested that smooth inhibition of the enzyme, not more than $25 \%$, is enough to restore the overactivated levels in the brain to the normal function and it is not too much to promote alternative physiological mechanism to compensate the $25 \%$ downactivity in other tissues with normal GSK-3 function.

Although two of the GSK-3 inhibitors which entered the clinical trials in the last five years failed in the first phases of development, it is clear that it was caused by the intrinsic toxicity of these two compounds related to their chemical structure, the potent inhibition on the enzyme, and the ATP competitiveness in their binding to GSK-3. In the meanwhile, NP-12 (tideglusib), the unique ATP noncompetitive GSK3 inhibitor that entered the clinical trials in May 2006, advances adequately in its long way to become an effective and accessible drug. Moreover, the great interest arising in GSK-3 inhibitors has led to the search for GSK-3 as a potential biomarker not only to follow the treatment progression but also to stratify the patients for better clinical trials design, and it could be possible to diagnose correctly and on time $\mathrm{AD}$ neuropathology [66]. All together locate GSK-3 inhibitors in the next horizon for AD treatment.

\section{Acknowledgments}

The authors gratefully acknowledge the financial support of Ministry of Science and Innovation MICINN (Project SAF2009-13015-C02-01) and Instituto de Salud Carlos III ISCiii Project no. RD07/0060/0015 (RETICS program). D. I. Perez acknowledges a postdoctoral fellowship from the CSIC (JAE program). There is no commercial conflict to declare.

\section{References}

[1] S. C. Marques, C. R. Oliveira, T. F. Outeiro, and C. M. Pereira, "Alzheimer's disease: the quest to understand complexity," The The Journal of Alzheimer's Disease, vol. 21, no. 2, pp. 373-383, 2010.

[2] J. Micheau and A. Marighetto, "Acetylcholine and memory: a long, complex and chaotic but still living relationship," Behavioural Brain Research, vol. 221, no. 2, pp. 424-429, 2011.

[3] E. Giacobini, "Cholinesterase inhibitors stabilize Alzheimer disease," Neurochemical Research, vol. 25, no. 9-10, pp. 11851190, 2000.

[4] M. Shanks, M. Kivipelto, R. Bullock, and R. Lane, "Cholinesterase inhibition: is there evidence for disease-modifying effects?" Current Medical Research and Opinion, vol. 25, no. 10, pp. 2439-2446, 2009.

[5] D. J. Selkoe, "Alzheimer's disease: genotypes, phenotype, and treatments," Science, vol. 275, no. 5300, pp. 630-631, 1997.

[6] R. A. Armstrong, "The molecular biology of senile plaques and neurofibrillary tangles in Alzheimer's disease," Folia Neuropathologica, vol. 47, no. 4, pp. 289-299, 2009.

[7] D. Saumier, A. Duong, D. Haine, D. Garceau, and J. Sampalis, "Domain-specific cognitive effects of tramiprosate in patients with mild to moderate Alzheimer's disease: ADAS-cog subscale results from the alphase study," Journal of Nutrition, Health \& Aging, vol. 13, no. 9, pp. 808-812, 2009.

[8] B. Vellas, "Tarenflurbil for Alzheimer's disease: a "shot on goal" that missed," The Lancet Neurology, vol. 9, no. 3, pp. 235237, 2010.

[9] N. F. Schor, "What the halted phase III $\gamma$-secretase inhibitor trial may (or may not) be telling us," Annals of Neurology, vol. 69, no. 2, pp. 237-239, 2011.

[10] K. Iqbal and I. Grundke-Iqbal, "Molecular mechanism of Alzheimer's neurofibrillary degeneration and therapeutic intervention," Annals of the New York Academy of Sciences, vol. 777, pp. 132-138, 1996.

[11] T. Del Ser, "Phase IIa clinical trial on Alzheimer's disease with NP12, a GSK-3 inhibitor," in Alzheimer's Dementia, vol 6, p S147, 2010.

[12] D. I. Perez, C. Gil, and A. Martinez, "Tau protein kinases inhibitors: from the bench to the clinical trials," in Emerging 
Drugs and Targets for Alzheimer's Disease, A. Martinez, Ed., vol. 1, pp. 173-194, Royal Society of Chemistry, 2010.

[13] F. Plattner, M. Angelo, and K. P. Giese, "The roles of cyclindependent kinase 5 and glycogen synthase kinase 3 in tau hyperphosphorylation," The Journal of Biological Chemistry, vol. 281, no. 35, pp. 25457-25465, 2006.

[14] C. Hooper, R. Killick, and S. Lovestone, "The GSK3 hypothesis of Alzheimer's disease," Journal of Neurochemistry, vol. 104, no. 6, pp. 1433-1439, 2008.

[15] S. M. de la Monte, M. Tong, N. Lester-Coll, M. Plater Jr., and J. R. Wands, "Therapeutic rescue of neurodegeneration in experimental type 3 diabetes: relevance to Alzheimer's disease," The Journal of Alzheimer's Disease, vol. 10, no. 1, pp. 89-109, 2006.

[16] A. Takashima, M. Murayama, O. Murayama et al., "Presenilin 1 associates with glycogen synthase kinase-3beta and its substrate tau," Proceedings of the National Academy of Sciences of the United States of America, vol. 95, no. 16, pp. 9637-9641, 1998.

[17] A. Takashima, "Drug development targeting the glycogen synthase kinase-3beta (GSK-3beta)-mediated signal transduction pathway: role of GSK-3beta in adult brain," Journal of Pharmacological Sciences, vol. 109, no. 2, pp. 174-178, 2009.

[18] H. Z. Wang, J. Brown, and M. Martin, "Glycogen synthase kinase 3: a point of convergence for the host inflammatory response," Cytokine, vol. 53, no. 2, pp. 130-140, 2011.

[19] K. Leroy, Z. Yilmaz, and J. P. Brion, "Increased level of active GSK-3beta in Alzheimer's disease and accumulation in argyrophilic grains and in neurones at different stages of neurofibrillary degeneration," Neuropathology and Applied Neurobiology, vol. 33, no. 1, pp. 43-55, 2007.

[20] A. Martinez and D. I. Perez, "GSK-3 inhibitors: a ray of hope for the treatment of Alzheimer's disease?" The Journal of Alzheimer's Disease, vol. 15, no. 2, pp. 181-191, 2008.

[21] E. M. Hur and F. Q. Zhou, "GSK3 signalling in neural development," Nature Reviews Neuroscience, vol. 11, no. 8, pp. 539-551, 2010.

[22] C. Xu, N. G. Kim, and B. M. Gumbiner, "Regulation of protein stability by GSK3 mediated phosphorylation," Cell Cycle, vol. 8, no. 24, pp. 4032-4039, 2009.

[23] M. Bouskila, M. F. Hirshman, J. Jensen, L. J. Goodyear, and K. Sakamoto, "Insulin promotes glycogen synthesis in the absence of GSK3 phosphorylation in skeletal muscle," American Journal of Physiology, vol. 294, no. 1, pp. E28-E35, 2008.

[24] S. E. Nikoulina, T. P. Ciaraldi, S. Mudaliar, P. Mohideen, L. Carter, and R. R. Henry, "Potential role of glycogen synthase kinase-3 in skeletal muscle insulin resistance of type 2 diabetes," Diabetes, vol. 49, no. 2, pp. 263-271, 2000.

[25] S. E. Nikoulina, T. P. Ciaraldi, S. Mudaliar, L. Carter, K. Johnson, and R. R. Henry, "Inhibition of glycogen synthase kinase 3 improves insulin action and glucose metabolism in human skeletal muscle," Diabetes, vol. 51, no. 7, pp. 21902198, 2002.

[26] J. R. Woodgett, "Glycogen synthase kinase 3: an introductory synopsis," in Glycogen Synthase Kinase 3 (GSK-3) and Its Inhibitors, A. Martinez, A. Castro, and M. Medina, Eds., pp. 3-23, John Wiley \& Sons, Hoboken, NJ, USA, 2006.

[27] D. Wu and W. Pan, "GSK3: a multifaceted kinase in Wnt signaling," Trends in Biochemical Sciences, vol. 35, no. 3, pp. 161-168, 2010.

[28] T. D. Gould, N. A. Gray, and H. K. Manji, "Effects of a glycogen synthase kinase-3 inhibitor, lithium, in adenomatous polyposis coli mutant mice," Pharmacological Research, vol. 48, no. 1, pp. 49-53, 2003.

[29] S. Phukan, V. S. Babu, A. Kannoji, R. Hariharan, and V. N. Balaji, "GSK3beta: role in therapeutic landscape and development of modulators," British Journal of Pharmacology, vol. 160 , no. 1 , pp. 1-19, 2010.

[30] A. Martinez, "Preclinical efficacy on GSK-3 inhibitors: towards a future generation of powerful drugs," Medicinal Research Reviews, vol. 28, no. 5, pp. 773-796, 2008.

[31] A. Martinez, A. Castro, I. Dorronsoro, and M. Alonso, "Glycogen synthase kinase 3 (GSK-3) inhibitors as new promising drugs for diabetes, neurodegeneration, cancer, and inflammation," Medicinal Research Reviews, vol. 22, no. 4, pp. 373-384, 2002.

[32] P. Bamborough, D. Drewry, G. Harper, G. K. Smith, and K. Schneider, "Assessment of chemical coverage of kinome space and its implications for kinase drug discovery," Journal of Medicinal Chemistry, vol. 51, no. 24, pp. 7898-7914, 2008.

[33] A. Martinez, M. Alonso, A. Castro, C. Perez, and F. J. Moreno, "First non-ATP competitive glycogen synthase kinase 3 beta (GSK-3beta) inhibitors: thiadiazolidinones (TDZD) as potential drugs for the treatment of Alzheimer's disease," Journal of Medicinal Chemistry, vol. 45, no. 6, pp. 1292-1299, 2002.

[34] L. Dugo, M. Collin, and C. Thiemermann, "Glycogen synthase kinase 3 beta as a target for the therapy of shock and inflammation," Shock, vol. 27, no. 2, pp. 113-123, 2007.

[35] M. L. Guzman, X. J. Li, C. A. Corbett et al., "Rapid and selective death of leukemia stem and progenitor cells induced by the compound 4-benzyl, 2-methyl, 1,2,4-thiadiazolidine, 3,5 dione (TDZD-8)," Blood, vol. 110, no. 13, pp. 4436-4444, 2007.

[36] M. Hamann, D. Alonso, E. Martin-Aparicio et al., "Glycogen synthase kinase-3 (GSK-3) inhibitory activity and structureactivity relationship (SAR) studies of the manzamine alkaloids. Potential for Alzheimer's disease," Journal of Natural Products, vol. 70, no. 9, pp. 1397-1405, 2007.

[37] D. Alonso and A. Martinez, "Marine compounds as a new source for glycogen synthase kinase 3 inhibitors," in Glycogen Synthase Kinase 3 (GSK-3) and Its Inhibitors, A. Martinez, A. Castro, and M. Medina, Eds., pp. 307-331, John Wiley \& Sons, Hoboken, NJ, USA, 2006.

[38] J. Peng, S. Kudrimoti, S. Prasanna et al., "Structure-activity relationship and mechanism of action studies of manzamine analogues for the control of neuroinflammation and cerebral infections," Journal of Medicinal Chemistry, vol. 53, no. 1, pp. 61-76, 2010

[39] G. Ermondi, G. Caron, I. G. Pintos et al., "An application of two MIFs-based tools (Volsurf+ and Pentacle) to binary QSAR: the case of a palinurin-related data set of non-ATP competitive Glycogen Synthase Kinase 3[beta] (GSK-3[beta]) inhibitors," European Journal of Medicinal Chemistry, vol. 46, no. 3, pp. 860-869, 2011.

[40] H. Eldar-Finkelman, A. Licht-Murava, S. Pietrokovski, and M. Eisenstein, "Substrate competitive GSK-3 inhibitors-strategy and implications," Biochimica et Biophysica Acta, vol. 1804, no. 3, pp. 598-603, 2010.

[41] H. Eldar-Finkelman and M. Eisenstein, "Peptide inhibitors targeting protein kinases," Current Pharmaceutical Design, vol. 15, no. 21, pp. 2463-2470, 2009.

[42] M. Shapira, A. Licht, A. Milman, C. G. Pick, E. Shohami, and H. Eldar-Finkelman, "Role of glycogen synthase kinase-3 beta in early depressive behavior induced by mild traumatic brain 
injury," Molecular and Cellular Neuroscience, vol. 34, no. 4, pp. 571-577, 2007.

[43] O. Kaidanovich-Beilin and H. Eldar-Finkelman, "Long-term treatment with novel glycogen synthase kinase-3 inhibitor improves glucose homeostasis in ob/ob mice: molecular characterization in liver and muscle," Journal of Pharmacology and Experimental Therapeutics, vol. 316, no. 1, pp. 17-24, 2006.

[44] C. P. Belani, "The role of irreversible EGFR inhibitors in the treatment of non-small cell lung cancer: overcoming resistance to reversible EGFR inhibitors," Cancer Investigation, vol. 28, no. 4, pp. 413-423, 2010.

[45] S. Conde, D. I. Perez, A. Martinez, C. Perez, and F. J. Moreno, "Thienyl and Phenyl alpha-halomethyl ketones: new inhibitors of glycogen synthase kinase (GSK-3 beta) from a library of compound searching," Journal of Medicinal Chemistry, vol. 46, no. 22, pp. 4631-4633, 2003.

[46] D. I. Perez, S. Conde, C. Perez et al., "Thienylhalomethylketones: irreversible glycogen synthase kinase 3 inhibitors as useful pharmacological tools," Bioorganic \& Medicinal Chemistry, vol. 17, no. 19, pp. 6914-6925, 2009.

[47] D. I. Pérez, V. Palomo, C. Pérez et al., "Switching reversibility to irreversibility in GSK-3 inhibitors: clues for specific design of new compounds," Journal of Medicinal Chemistry, vol. 54, no. 12, pp. 4042-4056, 2011.

[48] J. R. Woodgett, "Use of peptide substrates for affinity purification of protein-serine kinases," Analytical Biochemistry, vol. 180, no. 2, pp. 237-241, 1989.

[49] I. Hers, J. M. Tavare, and R. M. Denton, "The protein kinase C inhibitors bisindolylmaleimide I (GF 109203x) and IX (Ro 31-8220) are potent inhibitors of glycogen synthase kinase-3 activity," FEBS Letters, vol. 460, no. 3, pp. 433-436, 1999.

[50] A. Martinez, A. Castro, I. Cardelus, J. Llenas, and J. M. Palacios, "Arylimino-1,2,4-Thiadiazolidinones: a new family of potassium channel openers," Bioorganic \& Medicinal Chemistry, vol. 5, no. 7, pp. 1275-1283, 1997.

[51] A. Martinez, D. Alonso, A. Castro et al., "Synthesis and potential muscarinic receptor binding and antioxidant properties of 3-(thiadiazolyl)pyridine 1-oxide compounds," Archiv der Pharmazie, vol. 332, no. 6, pp. 191-194, 1999.

[52] A. Martinez, E. Fernandez, A. Castro et al., "N-benzylpiperidine derivatives of 1,2,4-thiadiazolidinone as new acetylcholinesterase inhibitors," European Journal of Medicinal Chemistry, vol. 35, no. 10, pp. 913-922, 2000.

[53] A. Martinez, A. Castro, C. Perez et al., "Heterocyclic inhibitors of glycogen synthase kinase 3," WO01/85685.

[54] H. C. Zhang, H. Ye, B. R. Conway et al., "3-(7-Azaindolyl)4-arylmaleimides as potent, selective inhibitors of glycogen synthase kinase-3," Bioorganic \& Medicinal Chemistry Letters, vol. 14, no. 12, pp. 3245-3250, 2004.

[55] T. A. Engler, S. Malhotra, T. P. Burkholder et al., "The development of potent and selective bisarylmaleimide GSK3 inhibitors," Bioorganic \& Medicinal Chemistry Letters, vol. 15, no. 4, pp. 899-903, 2005.

[56] A. Martinez, M. Alonso, A. Castro et al., "SAR and 3DQSAR studies on thiadiazolidinone derivatives: exploration of structural requirements for glycogen synthase kinase 3 inhibitors," Journal of Medicinal Chemistry, vol. 48, no. 23, pp. 7103-7112, 2005.

[57] A. Martinez, M. Alonso, A. Castro, and I. Dorronsoro, "GSK-3 inhibitors in Alzheimer's disease: TDZDs, from the discovery to clinical trials," in Medicinal Chemistry of Alzheimer Disease,
A. Martinez, Ed., pp. 225-253, Transworld Research Network, Kerala, India, 2008.

[58] http://www.noscira.com/prensa.cfm?mS=237\&mSS=616.

[59] http://clinicaltrials.gov/ct2/show/NCT01049399?term=tideglusib\&rank=1.

[60] R. Luna-Medina, M. Cortes-Canteli, S. Sanchez-Galiano et al., "NP031112, a thiadiazolidinone compound, prevents inflammation and neurodegeneration under excitotoxic conditions: potential therapeutic role in brain disorders," Journal of Neuroscience, vol. 27, no. 21, pp. 5766-5776, 2007.

[61] E. M. Ribe, M. Perez, B. Puig et al., "Accelerated amyloid deposition, neurofibrillary degeneration and neuronal loss in double mutant APP/tau transgenic mice," Neurobiology of Disease, vol. 20, no. 3, pp. 814-822, 2005.

[62] L. Sereno, M. Coma, M. Rodriguez et al., "A novel GSK-3 beta inhibitor reduces Alzheimer's pathology and rescues neuronal loss in vivo," Neurobiology of Disease, vol. 35, no. 3, pp. 359$367,2009$.

[63] M. Bolos, S. Fernandez, and I. Torres-Aleman, "Oral administration of a GSK3 inhibitor increases brain insulin-like growth factor I levels," The Journal of Biological Chemistry, vol. 285, no. 23, pp. 17693-17700, 2010.

[64] I. Torres-Aleman, "Targeting insulin-like growth factor-1 to treat Alzheimer's disease," Expert Opinion on Therapeutic Targets, vol. 11, no. 12, pp. 1535-1542, 2007.

[65] M. Medina and J. Avila, "Glycogen synthase kinase-3 (GSK-3) inhibitors for the treatment of Alzheimer's disease," Current Pharmaceutical Design, vol. 16, no. 25, pp. 2790-2798, 2010.

[66] A. Hye, F. Kerr, N. Archer et al., "Glycogen synthase kinase3 is increased in white cells early in Alzheimer's disease," Neuroscience Letters, vol. 373, no. 1, pp. 1-4, 2005. 


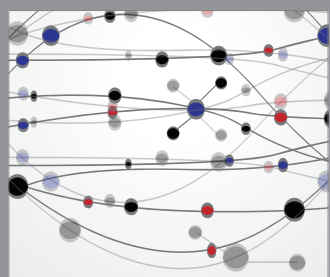

The Scientific World Journal
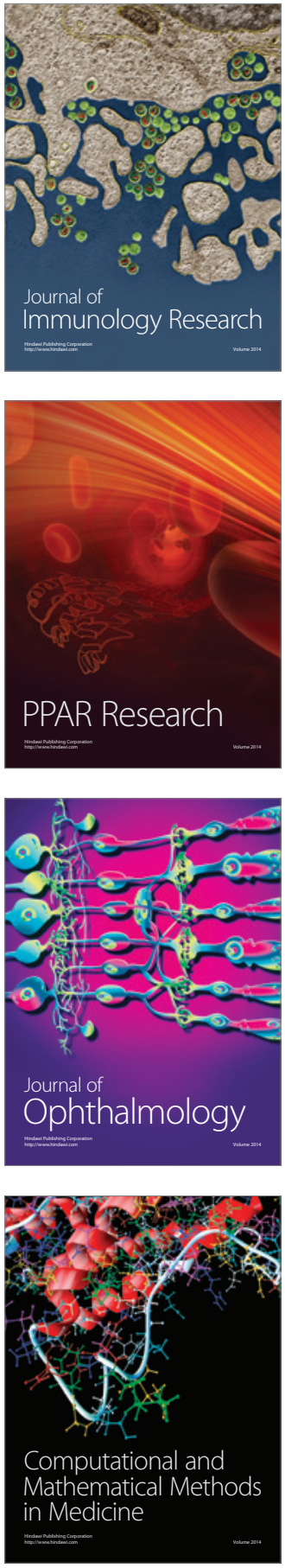

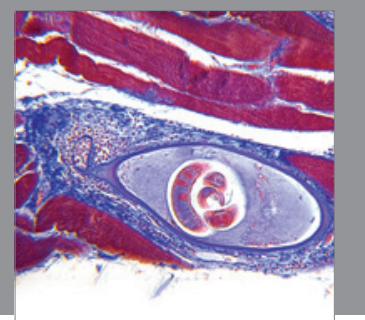

Gastroenterology

Research and Practice
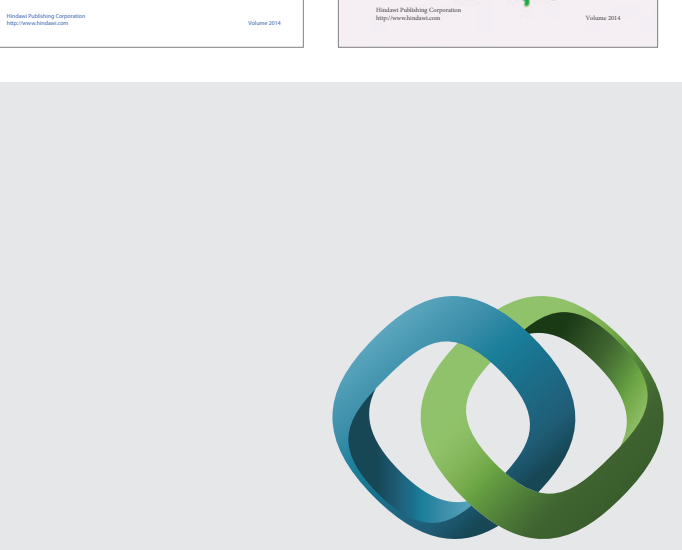

\section{Hindawi}

Submit your manuscripts at

http://www.hindawi.com
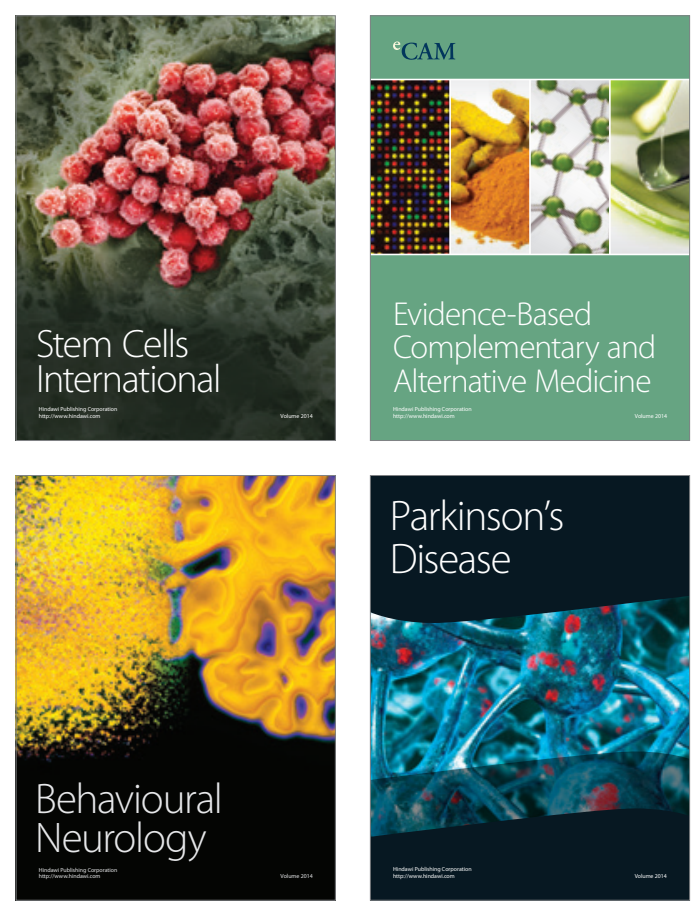

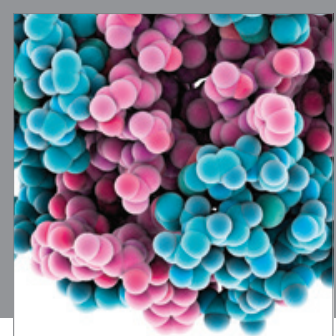

Journal of
Diabetes Research

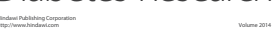

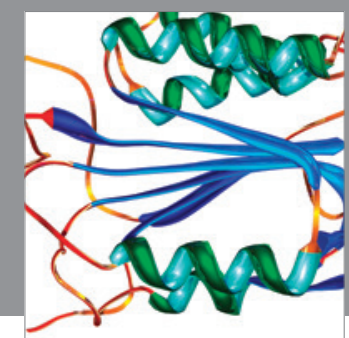

Disease Markers
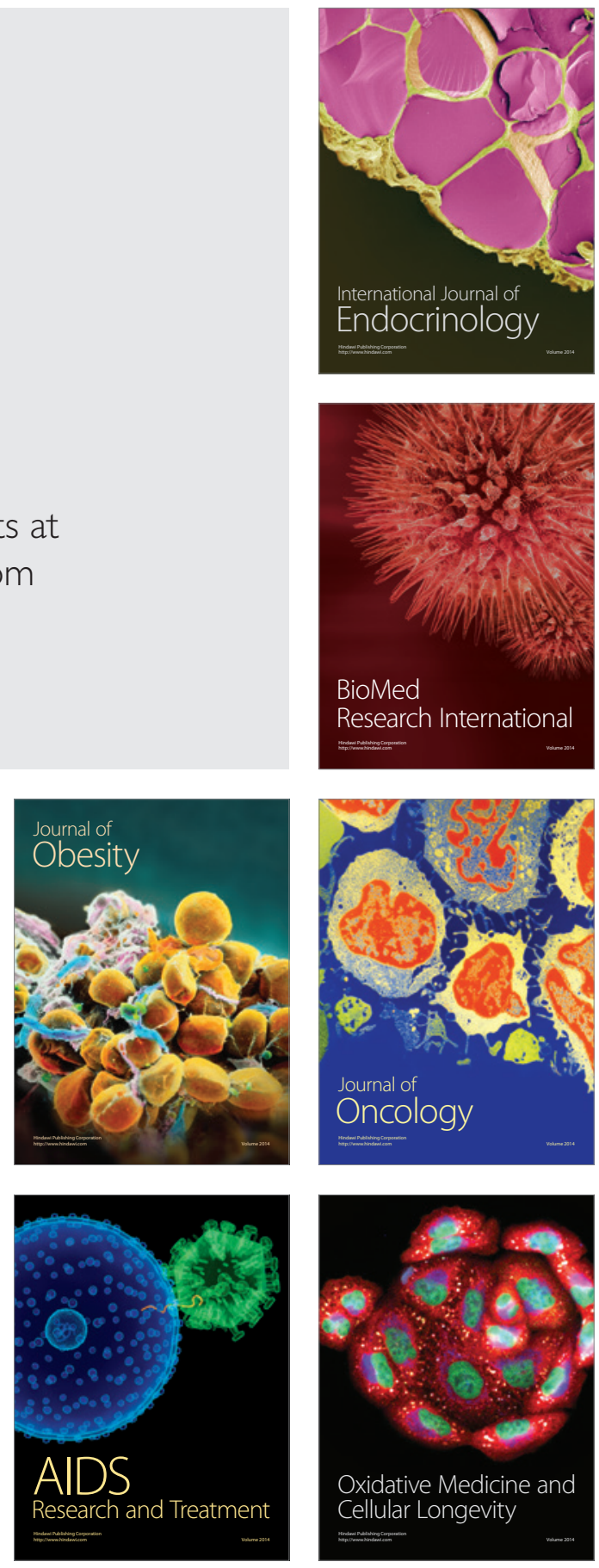